\title{
Toll-like receptor 3-induced immune response by poly(D,L-lactide-co-glycolide) nanoparticles for dendritic cell-based cancer immunotherapy
}

This article was published in the following Dove Press journal:

International Journal of Nanomedicine

2 November 2016

Number of times this article has been viewed

\author{
Hee Dong Han ${ }^{1} *$ \\ Yeongseon Byeon ${ }^{1, *}$ \\ Tae Heung Kang' \\ In Duk Jung' \\ Jeong-Won Lee ${ }^{2}$ \\ Byung Cheol Shin ${ }^{3}$ \\ Young Joo Lee ${ }^{4}$ \\ Anil K Sood ${ }^{5-7}$ \\ Yeong-Min Park' \\ 'Department of Immunology, School \\ of Medicine, Konkuk University, \\ Chungwondaero, Chungju-Si, \\ Chungcheongbuk-Do, ${ }^{2}$ Department of \\ Obstetrics and Gynecology, Samsung \\ Medical Center, Sungkyunkwan \\ University School of Medicine, Seoul, \\ ${ }^{3}$ Bio/Drug Discovery Division, Korea \\ Research Institute of Chemical \\ Technology, Yuseong-gu, Daejeon, \\ ${ }^{4}$ Department of Bioscience and \\ Biotechnology, Sejong University, \\ Kwang-Jin-Gu, Seoul, South Korea; \\ ${ }^{5}$ Department of Gynecologic \\ Oncology and Reproductive Medicine, \\ ${ }^{6}$ Department of Cancer Biology, \\ ${ }^{7}$ Center for RNA Interference and \\ Non-coding RNA, The University \\ of Texas MD Anderson Cancer \\ Center, TX, USA \\ *These authors contributed equally \\ to this work
}

Correspondence: Yeong-Min Park Department of Immunology, School of Medicine, Konkuk University, 268 Chungwondaero, Chungju-Si, Chungcheongbuk-Do 380-70I, South Korea

Tel +82 220496330

Fax +82 220496192

Email immun3023@kku.ac.kr

\begin{abstract}
Dendritic cells (DCs) are potent professional antigen-presenting cells that are capable of initiating a primary immune response and activating $\mathrm{T}$ cells, and they play a pivotal role in the immune responses of the host to cancer. Prior to antigen presentation, efficient antigen and adjuvant uptake by DCs is necessary to induce their maturation and cytokine generation. Nanoparticles (NPs) are capable of intracellular delivery of both antigen and adjuvant to DCs. Here, we developed an advanced poly(D,L-lactide-co-glycolide) (PLGA)-NP encapsulating both ovalbumin (OVA) as a model antigen and polyinosinic-polycytidylic acid sodium salt (Toll-like receptor 3 ligand) as an adjuvant to increase intracellular delivery and promote DC maturation. The PLGA-NPs were taken up by DCs, and their uptake greatly facilitated major histocompatibility class I antigen presentation in vitro. Moreover, vaccination with PLGA-NP-treated DCs led to the generation of ovalbumin-specific $\mathrm{CD} 8^{+} \mathrm{T}$ cells, and the resulting antitumor efficacy was significantly increased in EG.7 and TC-1 tumor-bearing mice compared to control mice $(P<0.01)$. Taken together, these findings demonstrated that the PLGA-NP platform may be an effective method for delivering tumor-specific antigens or adjuvants to DCs.
\end{abstract}

Keywords: cancer immunotherapy, PLGA nanoparticles, antigen delivery

\section{Introduction}

Multifunctional nanoparticles (NPs) have attracted interest in various biomedical fields for disease prevention and therapy. ${ }^{1,2}$ Several types of NPs have been used for the delivery of adjuvants and antigens to immune cells. These components can elicit strong immunotherapeutic responses to cancer and infectious diseases. In cancer immunotherapy, dendritic cell (DC)-based approaches hold great potential for antigen cross-presentation. ${ }^{3-5}$ DCs are the most effective antigen-presenting cells, which present antigens to CD8 ${ }^{+}$ $\mathrm{T}$ cells and secrete proinflammatory cytokines, resulting in tumor antigen-specific activation of $\mathrm{CD}^{+} \mathrm{T}$ cells that contribute to tumor inhibition. After internalizing antigen and adjuvant into DCs, DCs can exhibit antigen cross-presentation to cytotoxic T cells through extracellular major histocompatibility complex (MHC)-I molecules. ${ }^{6}$ Therefore, effective maturation of DCs is the key first step in DC-based cancer immunotherapy. Because antigen or adjuvant show limited penetration into DCs, efficient delivery systems are highly suitable for both antigen and adjuvant delivery into DCs. Recent studies have shown that antigen-loaded NPs increase DC maturation, enhancing antigen cross-presentation in DCs and inducing cytotoxic T cell responses. ${ }^{7}$

As DCs require high antigen uptake, various nanomaterials have been developed with specific physicochemical properties and are currently under extensive investigation 
for their potential utilization as drug delivery systems for immunotherapy ${ }^{8,9}$ and antitumor chemotherapeutic drugs. ${ }^{10,11}$ Poly(D,L-lactide-co-glycolide) (PLGA)-NPs, which are particularly attractive for clinical and biological applications because of their low toxicity, low immunogenicity, biocompatibility, and biodegradability, ${ }^{12,13}$ have been widely utilized as drug carriers in nanomedicine. ${ }^{14,15}$ These studies motivated us to know whether PLGA-NPs can increase the uptake efficiency of adjuvant or antigen by DCs and lead to an increase in antigen-specific $\mathrm{CD} 8^{+} \mathrm{T}$ cell responses.

Polyinosinic-polycytidylic acid (poly I:C) as an adjuvant promotes maturation of DCs through its interaction with Toll-like receptor 3 (TLR3), and could therefore improve antigen-mediated cross-presentation to antigen-specific $\mathrm{CD}^{+} \mathrm{T}$ cells. The potency of poly I:C has been demonstrated by its immunomodulatory effects in mice. ${ }^{16-18}$ Poly I:C mimics viral double-stranded RNA, which is a promising immunostimulatory candidate for vaccines directed against intracellular pathogens. However, TLR3 is localized in the endosome of DCs. Therefore, to activate TLR3, poly I:C has to penetrate the DCs. To overcome this limitation, a carrier system is needed to enhance intracellular delivery of poly I:C. Activation of TLR3 in DCs leads to the expression of proinflammatory cytokines such as interleukin (IL)-12, IL-6, and tumor necrosis factor-alpha (TNF- $\alpha$ ), resulting in enhanced innate and adaptive immune responses.

Here, we developed a PLGA-NP system encapsulating both ovalbumin (OVA) and poly I:C to increase the efficiency of their intracellular delivery into DCs and to promote DC maturation and antigen-specific cross-presentation (Figure 1). Taken together, our results demonstrated that PLGA-NPs were a highly efficient delivery system with therapeutic efficacy in EG.7 and TC-1 animal tumor models.

\section{Materials and methods Materials}

PLGA (Resomer RG502H, monomer ratio 50:50, molecular weight [MW] 10-12 kDa) was purchased from Boehringer Ingelheim (Ingelheim, Germany). Polyvinyl alcohol (80\% hydrolyzed, MW 9-10 kDa), chicken egg OVA, and poly I:C sodium salt were purchased from SigmaAldrich Co. (St Louis, MO, USA). Fluorescein isothiocyanate (FITC)-conjugated antimouse interferon (IFN)- $\gamma$, R-phycoerythrin (PE)-conjugated antimouse CD40, OVAspecific (SIINFEKL/H-2Kb) antibody, and mouse TNF- $\alpha$, IL-1 $\beta$, IL-6, IL-12p70 enzyme-linked immunosorbent assay (ELISA) Ready-SET-Go kit were purchased from eBioscience (San Diego, CA, USA). FITC-conjugated antimouse CD11c antibody, PE-conjugated antimouse CD8a, CD80, CD86, MHC class I, MHC class II, and mouse IFN- $\beta$ ELISA kit were purchased from Biolegend (San Diego, CA, USA). Roswell Park Memorial Institute Medium (RPMI) 1640 and fetal bovine serum (FBS) were purchased from Biowest (Nuaille, France). Granulocyte-macrophage colony-stimulating factor was purchased from JW CreaGene (Gyeonggi, South Korea). E7 peptide (MW 2.4 kDa, 42-64 amino acids, AGQAEPDRAHYNIVTFCCKCDS) was purchased from AnyGen Co. (Seoul, South Korea). Anti-CD8 antibody for $\mathrm{CD} 8^{+} \mathrm{T}$ cell depletion was purchased from BioXcell (West Lebanon, USA). All other materials were of analytical grade and used without further purification.

\section{Preparation of PLGA-NPs}

PLGA-NPs containing both OVA and poly I:C (PLGA [OVA + poly I:C]-NPs) were prepared by a water-in-oilin-water $(\mathrm{w} / \mathrm{o} / \mathrm{w})$ double emulsion solvent evaporation method. ${ }^{19,20}$ Briefly, $1 \mathrm{mg}$ OVA and $2 \mathrm{mg}$ poly I:C were

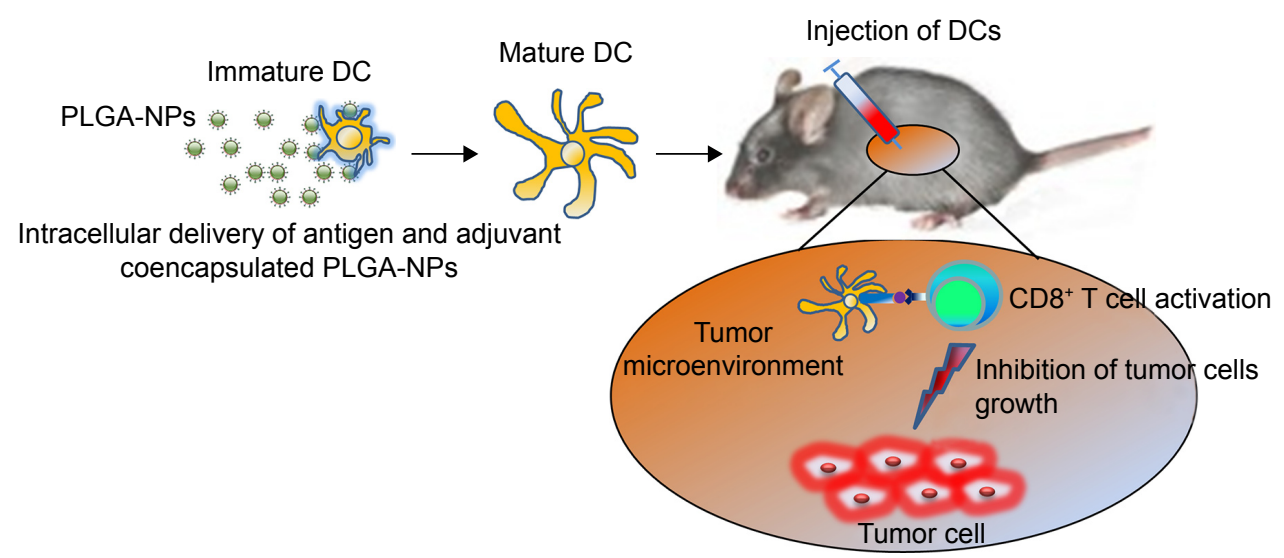

Figure I Schematic illustration of the overall concept of DC-based cancer immunotherapy

Notes: PLGA-NPs can be efficiently taken up by DCs. After uptake of PLGA-NPs, the PLGA-NPs prompt DC maturation and activation followed by release of adjuvant and antigen from NPs. Finally, mature DCs cross-present the antigen to active CD8 ${ }^{+} \mathrm{T}$ cells, which then kill the tumor cells.

Abbreviations: DC, dendritic cell; NPs, nanoparticles; PLGA, poly(D,L-lactide-co-glycolide). 
dissolved in $0.2 \mathrm{~mL}$ deionized water and mixed with $2 \mathrm{~mL}$ chloroform containing $40 \mathrm{mg}$ PLGA using a probetype sonicator at $4^{\circ} \mathrm{C}$ for $1 \mathrm{~min}$ (20 pulses of $5 \mathrm{~s}$ with a $3 \mathrm{~s}$ gap). The primary emulsion was further emulsified with a secondary aqueous phase $(10 \mathrm{~mL}$ of $2.0 \% \mathrm{w} / \mathrm{v}$ polyvinyl alcohol) at $4^{\circ} \mathrm{C}$ for $10 \mathrm{~min}$ to form a secondary emulsion. To remove chloroform completely, the emulsion was evaporated using a rotary evaporator at $30^{\circ} \mathrm{C}$ under vacuum. After evaporation, the suspension of PLGA-NPs was washed three times with deionized water at $4^{\circ} \mathrm{C}$ by centrifugation at $13,000 \mathrm{rpm}$ for $20 \mathrm{~min}$. To quantify encapsulated OVA or poly I:C, the PLGA-NPs were dissolved in $0.1 \mathrm{M} \mathrm{NaOH}$ and $0.1 \%$ sodium dodecyl sulfate, incubated at $27^{\circ} \mathrm{C}$ overnight, and then measured using a bicinchoinic acid protein assay kit (Pierce Biotechnology, Rockford, IL, USA) for OVA or a NanoDrop 2,000 spectrophotometer (Thermo Fisher Scientific, Waltham, MA, USA) for poly I:C absorbance at $260 \mathrm{~nm}$ in ultraviolet light. ${ }^{21}$ The size and surface charge of the PLGA-NPs were analyzed by dynamic light scattering using an electrophoretic light scattering photometer (ELS-Z; Otsuka Electronics, Osaka, Japan). In addition, the morphology of PLGA-NPs was monitored using scanning electron microscopy (Mira 3 LMU FEG; Tescan, Brno, Czech Republic).

\section{Mice and cell lines}

Female C57BL/6 mice (5-6 weeks old, 20 g) were purchased from ORIENT (Gapyeong, South Korea) and maintained under a protocol approved by the Konkuk University Institutional Animal Care and Use Committee (Ref No: KU14157). All procedures were performed according to approved protocols and were in accordance with recommendations for the proper care and use of animals at the specific pathogen-free housing facility at Konkuk University. In this study, we used 168 mice for in vivo cytotoxic $\mathrm{CD}^{+} \mathrm{T}$ cell activation (30 mice), cytotoxic T lymphocyte (CTL) assays (30 mice), therapeutic efficacy tests for the EG.7 and TC-1 tumor model (72 mice), and therapeutic efficacy with $\mathrm{CD}^{+} \mathrm{T}$ cell depletion (36 mice). OVA expressing EG.7 cells (EL4 cell line transfected with the gene encoding OVA) and TC-1 cells expressing HPV16 and HPV-E7 proteins were cultured in RPMI 1640 medium supplemented with $0.1 \%$ gentamycin and $10 \%$ FBS.

\section{Generation of DCs from mouse bone marrow}

DCs were harvested from the bone marrow of C57BL/6 mice. ${ }^{22}$ Briefly, bone marrow was collected from the tibias and femurs. Red blood cells were depleted using $0.83 \mathrm{M}$ $\mathrm{NH}_{4} \mathrm{Cl}$ buffer (Sigma-Aldrich Co.). The bone marrow cells $\left(2 \times 10^{6}\right.$ cells/well $)$ were collected and cultured in a six-well culture plate containing $6 \mathrm{~mL}$ culture medium supplemented with $10 \% \mathrm{FBS}, 0.1 \%$ gentamycin, and $20 \mathrm{ng} / \mathrm{mL}$ mouse recombinant granulocyte-macrophage colony-stimulating factor.

\section{Intracellular uptake assay}

Before testing for intracellular uptake of PLGA-NPs, we first conjugated tetramethylrhodamine with OVA or FITC with poly I:C for flow cytometric and confocal microscopic analyses, respectively. Briefly, the DCs were incubated with PLGA-NPs including OVA and poly I:C for $30 \mathrm{~min}$ at $37^{\circ} \mathrm{C}$. After incubation, PLGA-NP uptake by the DCs was analyzed by flow cytometry (FACSCalibur with CELLQuest software; BD Biosciences, Franklin Lakes, NJ, USA) and confocal microscopy (DeltaVision; GE Healthcare Bio-Sciences Corp., Piscataway, NJ, USA).

\section{Cell viability}

The viability of cells treated with PLGA-NPs was determined using 3-(4,5-dimethylthiazol-2-yl)-2,5-diphenyltetrazolium bromide assay. ${ }^{23}$ DCs were transferred to 96 -well plates at $5 \times 10^{4}$ cells per well and incubated in a $5 \% \mathrm{CO}_{2}$ incubator overnight to allow the cells to attach. PLGA-NPs were added to the wells, and the cells were incubated for an additional 24 or 48 hr. Absorbance was read using a microplate reader (EL808; Bio-Tek, Winooski, VT, USA) at $590 \mathrm{~nm}$. The means and standard deviations of triplicates were calculated.

\section{DC maturation and cytokine generation}

To confirm DC maturation, DCs were cultured in 6-well plates at a density of $2 \times 10^{6}$ cells per well and allowed to adhere overnight. DCs alone as a control, PLGA-NPs, PLGA (OVA)NPs, PLGA (poly I:C)-NPs, or PLGA (OVA + poly I:C)-NPs containing OVA $(100 \mu \mathrm{g} / \mathrm{mL})$ and poly I:C $(100 \mu \mathrm{g} / \mathrm{mL})$ were added to the wells. After $48 \mathrm{hr}$ of incubation, DC maturation was analyzed by flow cytometry. DCs were stained with FITC-conjugated anti-CD11c and PE-conjugated antiCD40, anti-CD80, anti-CD86, anti-MHC class I, anti-MHC class II, and anti-OVA-specific (SIINFEKL/H-2Kb) MHC class I antibodies. In addition, proinflammatory cytokines (IL-1 $\beta$, IL-6, IL-12p70, and TNF- $\alpha$ ) secreted from DCs during maturation were analyzed using cytokine-specific ELISA kit (eBioscience).

\section{In vivo $C D 8^{+} T$ cell activation}

To determine $\mathrm{CD} 8^{+} \mathrm{T}$ cell activation, DCs treated with PLGANPs were injected subcutaneously (sc) into C57BL6 mice (five mice/group). Mice were monitored daily for adverse effects of vaccination and were sacrificed at 7 days after 
the last immunization (two times weekly). DCs treated with PLGA-NPs were administered in the right flank region with (1) DCs only as a control, (2) DCs treated with OVA solution, (3) DCs treated with PLGA-NPs, (4) DCs treated with PLGA (OVA)-NPs, (5) DCs treated with PLGA (poly I:C)-NPs, or (6) DCs treated with PLGA (OVA + poly I:C)-NPs. At 7 days after the last immunization, $1 \times 10^{7}$ splenocytes were harvested from the vaccinated mice, suspended in $1 \mathrm{~mL}$ of RPMI with $10 \%$ FBS, $0.1 \%$ gentamycin, and $0.5 \%$ 2-mercaptoethanol, and incubated for $16 \mathrm{hr}$ with GolgiPlug (BD Biosciences) and OVA peptide $(1 \mu \mathrm{g} / \mathrm{mL}) \cdot{ }^{24,25}$ Cells were washed, stained with PE-conjugated anti-CD8a antibody, fixed, permeabilized, and stained with FITC-conjugated anti-IFN- $\gamma$ antibody. Cells were analyzed by flow cytometry.

\section{In vivo $C T L$ assay}

For CTL assays, DCs treated with PLGA-NPs $\left(2 \times 10^{6}\right.$ cells/ mouse) were injected sc three times, once per week, into C57BL6 mice. ${ }^{26}$ Four groups (five mice/group) were immunized in the right flank region with (1) DCs only as a control, (2) DCs treated with OVA solution, (3) DCs treated with PLGA-NPs, (4) DCs treated with PLGA (OVA)-NPs, (5) DCs treated with PLGA (poly I:C)-NPs, or (6) DCs treated with PLGA (OVA + poly I:C)-NPs. At 7 days after the last immunization (two times weekly), the splenocytes were harvested and pulsed with or without OVA peptide $(10 \mu \mathrm{g} / \mathrm{mL})$ for $45 \mathrm{~min}$. The OVA peptide-pulsed or unpulsed populations were then incubated with either $10 \mu \mathrm{M}$ (high) or $1 \mu \mathrm{M}$ (low) carboxyfluorescein succinimidyl ester (CFSE) at $37^{\circ} \mathrm{C}$ for $10 \mathrm{~min}$. The two populations were then mixed at a 1:1 ratio. Finally, the mixed splenocytes were injected ( $10^{7}$ cells/mouse) intravenously through the tail vein into the immunized C57BL6 mice. After $18 \mathrm{hr}$, the splenocytes were isolated and the numbers of $\mathrm{CFSE}^{\text {high }}$ and $\mathrm{CFSE}^{\text {low }}$ cells were measured by flow cytometry.

\section{Antitumor efficacy of DCs treated with PLGA-NPs}

To produce tumors, EG. 7 lymphoma cells $\left(1 \times 10^{6}\right.$ cells per 0.1 mL Hank's Balanced Salt Solution [HBSS]) or TC-1 cervical tumor cells $\left(1 \times 10^{6}\right.$ cells per $0.1 \mathrm{~mL}$ HBSS $)$ were injected sc into mice. We selected EG.7 tumor cells, which express OVA peptide, ${ }^{27}$ and TC-1 tumor cells, which express HPV16 E7 protein. ${ }^{28}$ Therefore, EG.7 and TC-1 tumor models would exhibit antigen-specific immune responses against cytotoxic $\mathrm{CD}^{+} \mathrm{T}$ cells, resulting in antigen-specific immune responses to EG.7 (OVA specific) and TC-1 (E7 specific) cells. One week following injection, the mice were randomly allocated into the following groups ( $\mathrm{n}=6$ mice per group): (1) DCs only as a control, (2) DCs treated with OVA solution, (3) DCs treated with PLGA-NPs, (4) DCs treated with PLGA (OVA)NPs, (5) DCs treated with PLGA (poly I:C)-NPs, and (6) DCs treated with PLGA (OVA + poly I:C)-NPs. DCs were administered three times ( $\mathrm{sc}, 2 \times 10^{6}$ cells/mouse, 1 week intervals for 3 weeks) in the right flank. Tumor volume and survival were recorded. Tumors were measured using calipers, and the tumor volume was calculated using the following formula: tumor volume $\left(\mathrm{mm}^{3}\right)=$ length $\times(\text { width })^{2} / 2$. In addition, we confirmed $\mathrm{CD}^{+} \mathrm{T}$ cell localization using anti-CD8 antibody and tumor cell apoptosis using terminal deoxynucleotidyl transferase dUTP nick-end labeling assays in the tumor tissue by immunohistochemical staining analysis, as described previously using formalin-fixed, paraffin-embedded EG.7 and TC-1 tumor tissue specimens. ${ }^{29}$ The analysis was recorded in five random fields for each slide and was performed by two investigators in a blinded fashion.

In addition, we performed in vivo antibody depletion assay to confirm $\mathrm{CD}^{+} \mathrm{T}$ cell-mediated antitumor therapeutic efficacy. ${ }^{27,30}$ We used monoclonal antibody 2.43 for $\mathrm{CD}^{+}$ $\mathrm{T}$ cell depletion. One week after tumor cell injection, the mice were randomly allocated into the following groups $(n=6$ mice per group): (1) DCs only as a control, (2) DCs treated with PLGA (OVA + poly I:C)-NPs with $\mathrm{CD}^{+} \mathrm{T}$ cell depletion, and (3) DCs treated with PLGA (OVA)-NPs. DCs were administered three times ( $\mathrm{sc}, 2 \times 10^{6}$ cells/mouse, 1 week intervals for 3 weeks) in the right flank. Depletions (100 $\mu \mathrm{g}$ of anti-CD8 antibody, once daily) were initiated 5 days after tumor cell injection and lasted till the end of the experimental period. The completeness of depletion was confirmed by flow cytometry analysis. More than 90\%-95\% depletion of the appropriate subset was achieved with normal levels of the other lymphocyte subsets. Tumor was measured using calipers, and the tumor volume was calculated with the above equation.

\section{Statistical analysis}

Differences in continuous variables were analyzed using Student's $t$-test for comparing two groups. Nonparametric one-way or two-way analysis of variance was performed with Statistical Package for the Social Sciences (SPSS, Inc.), which was used for all statistical analyses. Results with $P$-value of $<0.05$ were considered statistically significant.

\section{Results \\ Characteristics of PLGA-NPs}

In this study, we selected PLGA as the polymer matrix because it is particularly attractive for clinical and biological 
A

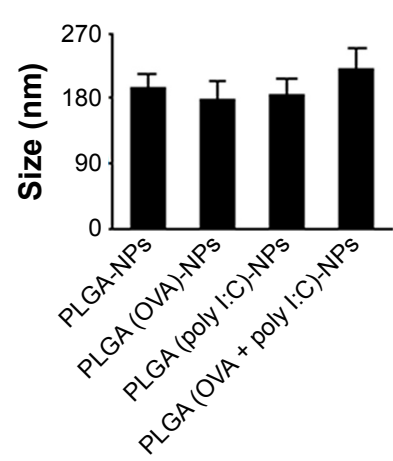

B

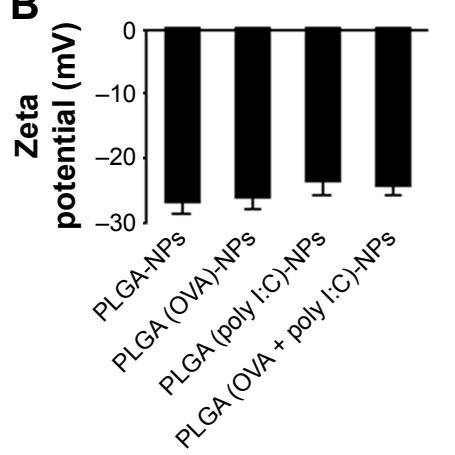

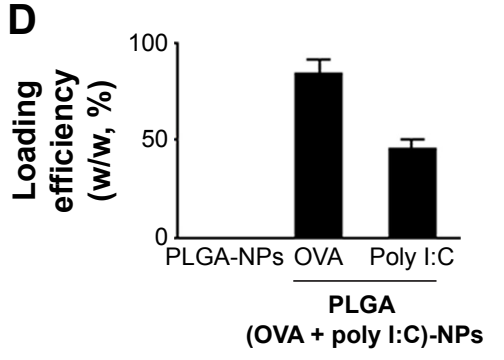

C

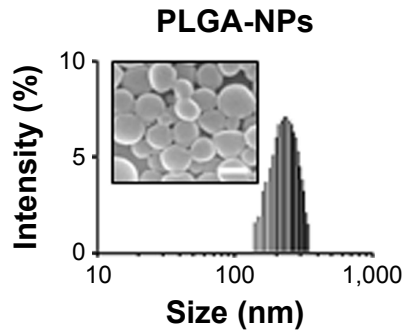

PLGA (OVA)-NPs

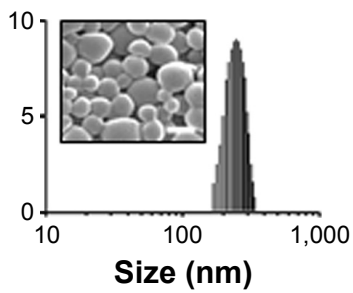

PLGA (poly I:C)-NPs

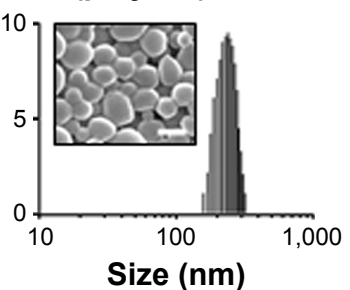

PLGA
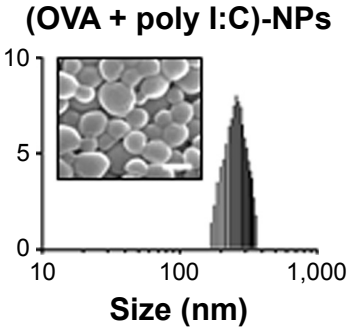

Figure 2 Physical properties of PLGA-NPs.

Notes: (A) Size and (B) zeta potential of PLGA-NPs were measured by light scattering with a particle size analyzer and Zeta Plus, respectively. (C) Histograms of various PLGA-NPs showing the size distribution and morphology of PLGA-NPs by scanning electron microscopy. Scale bar: $200 \mathrm{~nm}$. (D) Loading efficiency of OVA and poly I:C into PLGA-NPs was determined by BCA protein assay. The data are presented as the mean $\pm S D(n=3)$.

Abbreviations: NPs, nanoparticles; OVA, ovalbumin; PLGA, poly(D,L-lactide-co-glycolide); poly I:C, polyinosinic-polycytidylic acid; SD, standard deviation.

applications due to its low toxicity, biocompatibility, biodegradability, and low immunogenicity. ${ }^{12}$ Here, we successfully prepared PLGA-NPs using a w/o/w double emulsion method to encapsulate both OVA and poly I:C. We first confirmed the physical properties of PLGA-NPs, PLGA (OVA)-NPs, PLGA (poly I:C)-NPs, and PLGA (OVA + poly I:C)-NPs (Figure 2). The mean particle size and zeta potential were around $200 \pm 3.69 \mathrm{~nm}$ and $-30 \mathrm{mV}$, respectively (Figure 2A and B). Representative histograms of the particle size distribution are shown in Figure 2C. These data indicated that the encapsulation of either OVA or poly I:C did not affect the formation or physicochemical properties of these PLGA-NP formulations. Additionally, the morphology of these PLGA-NPs was confirmed by scanning electron microscopy. The NPs were spherical and the size was $200 \mathrm{~nm}$ (Figure 2C). The loading efficiency of OVA and poly I:C was $90 \%$ and $50 \%$, respectively (Figure 2D). In addition, we also confirmed the release of OVA from PLGA (OVA + poly I:C)-NPs, indicating that OVA was easily released in an acidic environment at $37^{\circ} \mathrm{C}$ (Figure $\mathrm{S} 1$ ).

\section{Intracellular uptake of PLGA-NPs in DCs}

We next assessed the intracellular uptake of PLGA (OVA + poly I:C)-NPs by flow cytometry and confocal microscopy (Figures 3 and S2). Flow cytometric analysis indicated that the PLGA-NPs exhibited highly efficient and dosedependent intracellular delivery compared with control and OVA solution (Figure 3A). In addition, confocal microscopic analysis (Figure 3B) showed that uptake of PLGA (OVA + poly I:C)-NPs by DCs was consistent with the flow cytometric data.

\section{Cell viability of PLGA-NPs}

To assess the possible cytotoxic effect of PLGA-NP formulations on DCs, we tested the viability of DCs after uptake of PLGA-NPs with (Figure 4A) or without encapsulated OVA or poly I:C (Figure 4B). As shown in Figure 4A and B, cell viability was as high as $90 \%$, even with increasing PLGA concentration, indicating that the base PLGA-NP composition was not appreciably toxic to the DCs.

\section{DC maturation and activation after uptake of PLGA-NPs}

To assess maturation and activation, we characterized the presence of activation markers and proinflammatory cytokine expression in DCs. DCs were isolated from the bone marrow of mice and incubated with or without PLGA-NPs (100 $\mu \mathrm{g}$ poly I:C). Various maturation and activation markers were then assessed using flow cytometry and ELISA. DCs treated with PLGA (OVA + poly I:C)-NPs expressed significantly 
A

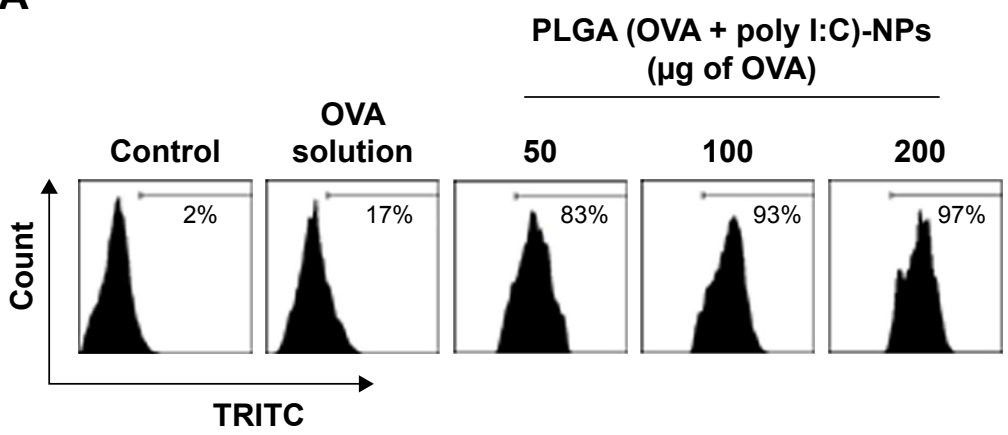

TRITC

\section{B}
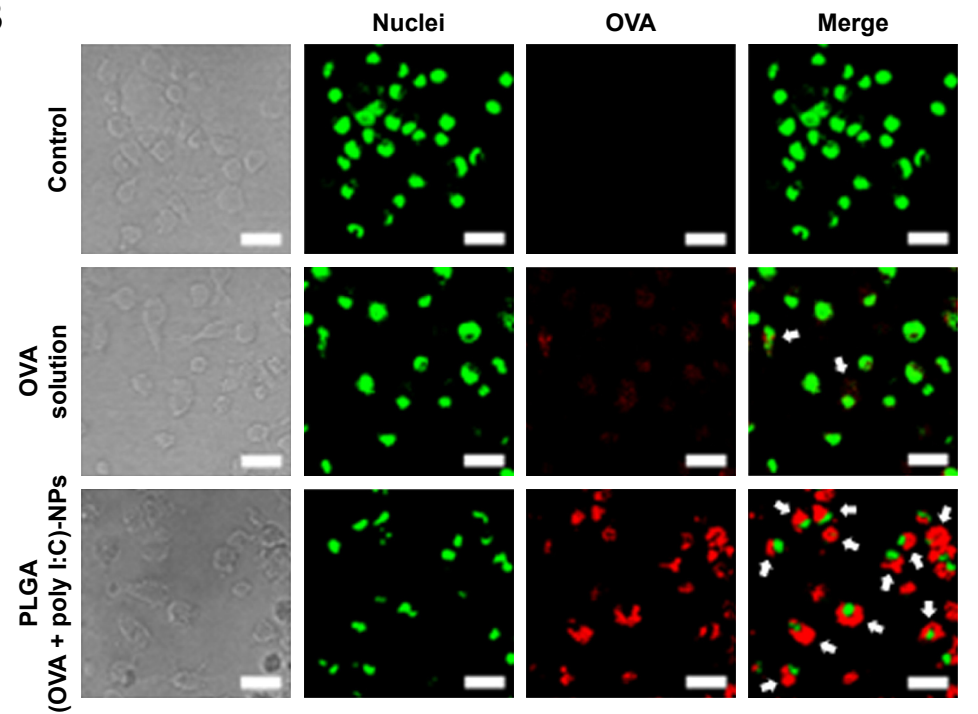

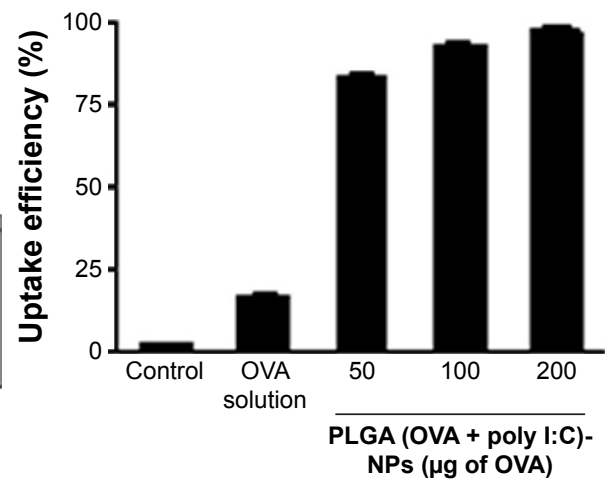

Figure 3 Intracellular uptake of PLGA-NPs in DCs.

Notes: (A) Intracellular delivery efficiency of PLGA-NPs was evaluated by flow cytometry. The cells were incubated with PLGA-NPs encapsulating TRITC-labeled OVA for 30 min at $37^{\circ} \mathrm{C}$. (B) Fluorescence images were obtained by confocal microscopy. Red color shows TRITC-labeled OVA and green color shows the nuclei. Scale bar: $20 \mu \mathrm{m}$. $* P$-value (PLGA [OVA + poly I:C]-NPs) vs control. $P<0.05$.

Abbreviations: DCs, dendritic cells; NPs, nanoparticles; OVA, ovalbumin; PLGA, poly(D,L-lactide-co-glycolide); poly I:C, polyinosinic-polycytidylic acid; TRITC, tetramethylrhodamine.

higher levels of the maturation surface markers CD40, CD80, CD86, MHC class I, and MHC class II compared to control DCs, which exhibited expression levels similar to those treated with empty PLGA-NPs as a negative control (Figure 5A). These results indicated that PLGA (OVA + poly I:C)-NPs activated and induced maturation of DCs. We also confirmed OVA-specific MHC I-mediated antigen cross-presentation. As shown in Figure 5A, OVAdependent MHC I expression was significantly higher than that in control DCs (Figure 5A). In addition, incubation with PLGA (OVA + poly I:C)-NPs significantly increased the expression of proinflammatory cytokines IL-1 $\beta$, IL-6, IL-12p70, and TNF- $\alpha$ during DC maturation relative to that in control DCs (Figure 5B). Collectively, these data demonstrated that PLGA (OVA + poly I:C)-NPs could elicit maturation, activation, and induce antigen-specific cross-presentation in DCs.

\section{In vivo antigen-specific $C D 8^{+} T$ cell activation and CTL assay}

We next evaluated the potential of PLGA (OVA + poly I:C)-NPs to generate antigen-specific adaptive cellular immune responses using PLGA (OVA + poly I:C)-NPs activated DCs as a DC-based vaccination. C57BL/6 mice (five per group) were immunized with DCs treated with PLGA-NPs $\left(2 \times 10^{6}\right)$ pulsed with or without OVA peptide $(1 \mu \mathrm{g} / \mathrm{mL})$, and splenocytes were harvested and analyzed by flow cytometry 7 days after the last immunization. Mice vaccinated with PLGA (OVA + poly I:C)-NP-treated DCs pulsed with OVA peptides generated significantly higher numbers of activated $\mathrm{CD} 8^{+} \mathrm{T}$ cells as measured by IFN- $\gamma$ secretion (Figure $6 \mathrm{~A}$ ). This result indicated that PLGA (OVA + poly I:C)-NP-treated DCs could activate antigen-specific $\mathrm{CD} 8^{+} \mathrm{T}$ cells, which were capable of tumor protection. 


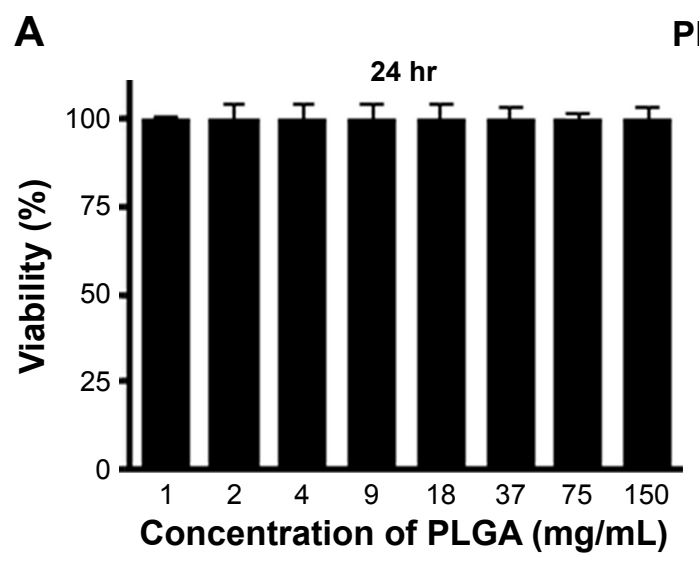

PLGA-NPs
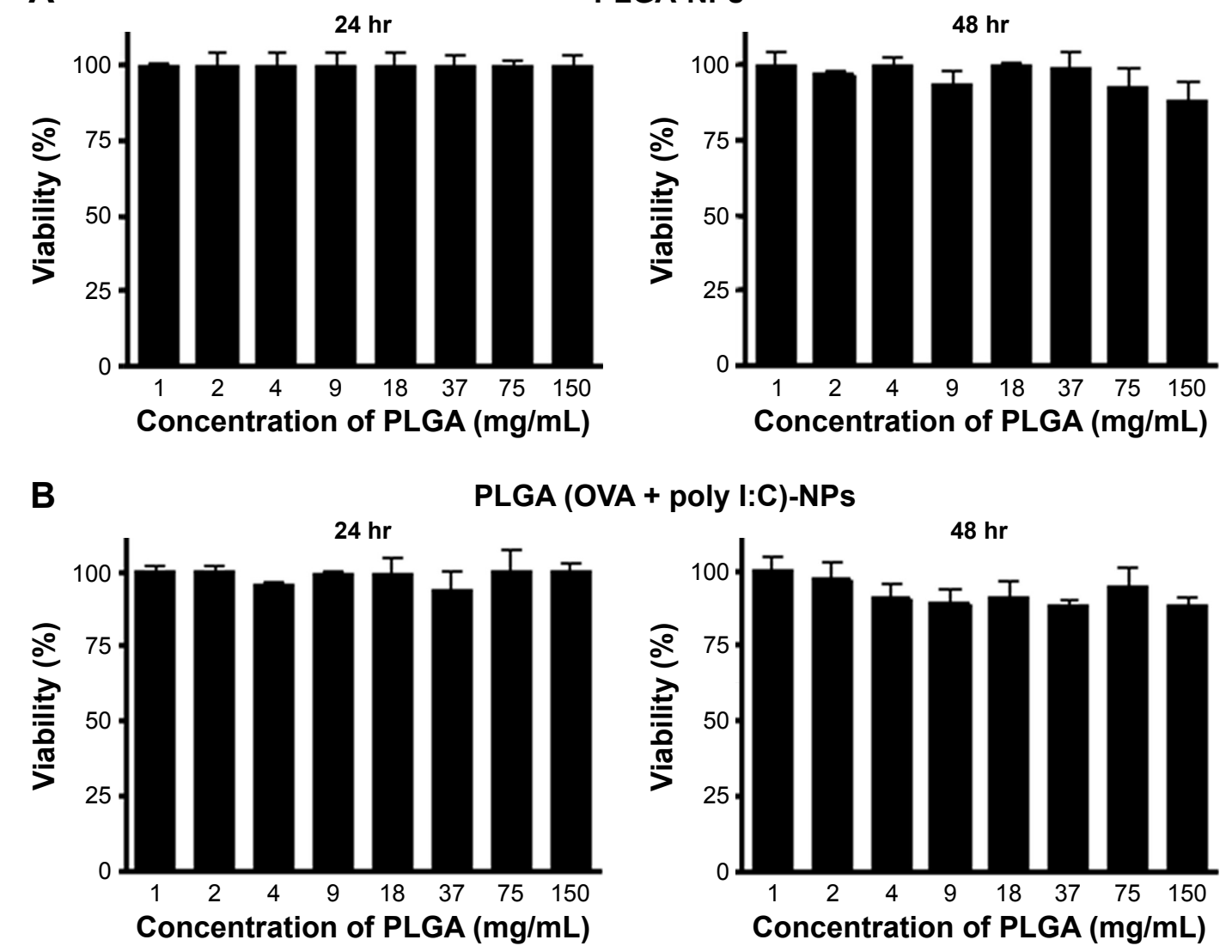

Figure 4 Viability of DCs after uptake of (A) PLGA-NPs and (B) PLGA (OVA + poly I:C)-NPs at different time periods (24 and 48 hr).

Notes: MTT assay was performed to determine cell viability following treatment with indicated concentrations of PLGA-NPs. The data are represented as mean \pm SD ( $n=3$ ). Abbreviations: DCs, dendritic cells; MTT, 3-(4,5-dimethylthiazol-2-yl)-2,5-diphenyltetrazolium bromide; NPs, nanoparticles; OVA, ovalbumin; PLGA, poly(D,L-lactide-coglycolide); poly I:C, polyinosinic-polycytidylic acid; SD, standard deviation.

In addition, we confirmed the ability of activated $\mathrm{CD} 8^{+}$ $\mathrm{T}$ cells stimulated by DCs treated with PLGA (OVA + poly I:C)-NPs to kill tumor cells using an in vivo CTL activity assay. C57BL/6 mice (five per group) were immunized with DCs treated with PLGA-NPs $\left(2 \times 10^{6}\right)$ pulsed with or without OVA peptide $(1 \mu \mathrm{g} / \mathrm{mL})$, and splenocytes were harvested and analyzed by flow cytometry 7 days after the last immunization. We observed significantly higher target cell lysis in mice that received PLGA (OVA + poly I:C)-NP-treated DCs than that in mice immunized with DCs only, DCs treated with PLGA-NPs, or DCs treated with OVA solution (Figure 6B). These results indicated that PLGA (OVA + poly I:C)-NP-treated DCs activated cytotoxic $\mathrm{CD} 8^{+} \mathrm{T}$ cells, and thus served as a cancer vaccine against OVA-positive tumor cells by enhancing cytotoxic activity.

\section{Therapeutic efficacy of vaccination with PLGA-NP-treated DCs}

To determine the potential therapeutic efficacy of the PLGANPs, we selected EG.7-OVA cells, which are an attractive tumor model for OVA-based experimental models of anticancer vaccines. Seven days following the sc injection of tumor cells, mice were randomly allocated to the following groups ( $\mathrm{n}=6$ mice/group): (1) DCs only, (2) DCs treated with OVA solution, (3) DCs treated with PLGA-NPs, (4) DCs treated with PLGA (OVA)-NPs, (5) DCs treated with PLGA (poly I:C)-NPs, and (6) DCs treated with PLGA (OVA + poly I:C)NPs. DCs were injected sc 7 days after tumor inoculation three times at 1 week intervals (Figure 7A). DCs treated with PLGA (OVA + poly I:C)-NPs showed significantly higher inhibition of tumor growth compared to DCs alone (control group, $93 \%$ reduction; $P<0.005$ ), OVA solution ( $90 \%$ reduction; $P<0.007$ ), and PLGA-NPs (93\% reduction; $P<0.004$ ) (Figure 7B and D). Notably, $100 \%$ of mice vaccinated with PLGA (OVA + poly I:C)-NPs-treated DCs survived for up to 45 days, while mice in the other vaccinated groups died within 45 days after tumor inoculation (Figure 7C). The data suggested that vaccination with PLGA (OVA + poly I:C)NP-treated DCs could enhance therapeutic antitumor efficacy and prolong survival. There were no differences in total body 


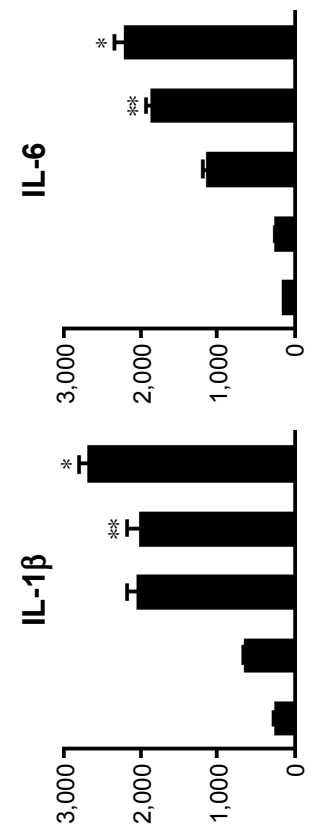

(7u/6d)

m иo!̣eגұ๐əoиоo
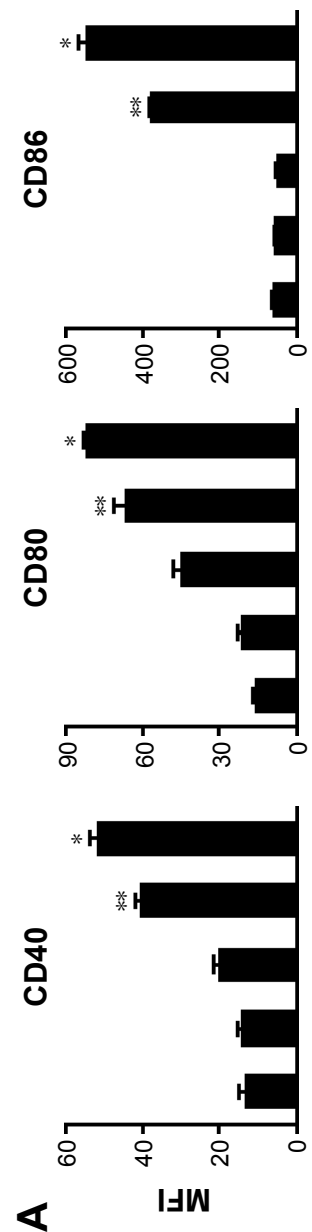
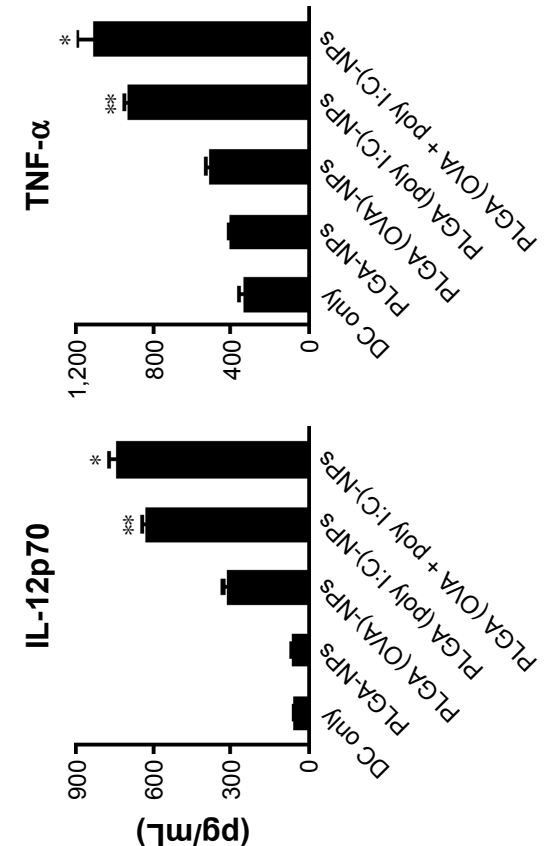

uo!̣eגұ๐əouоo
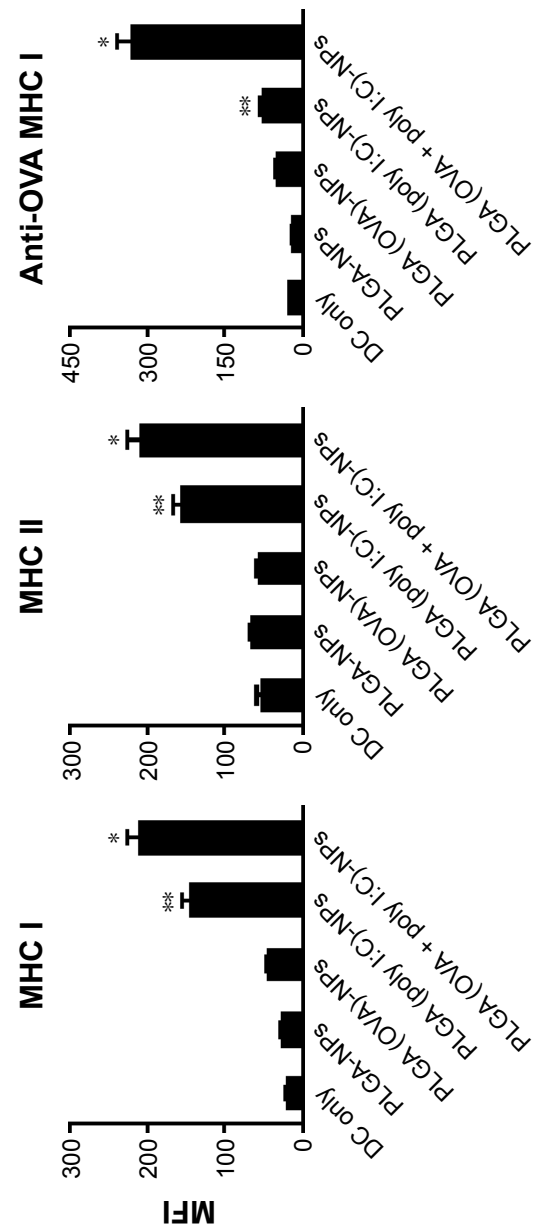

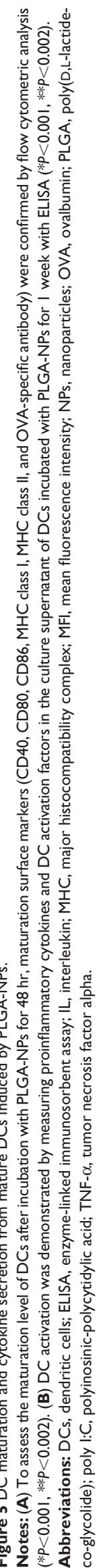


A

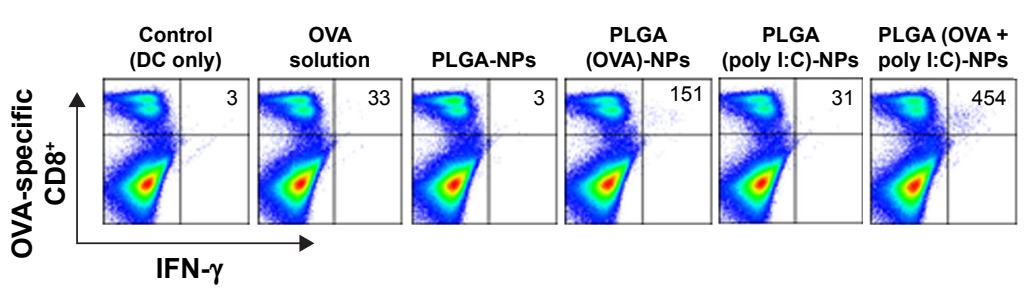

B

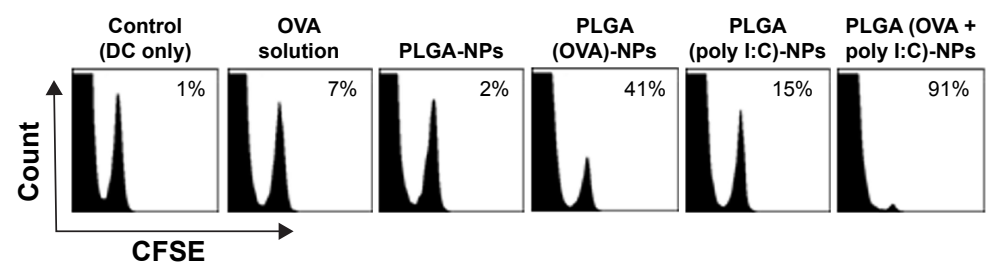

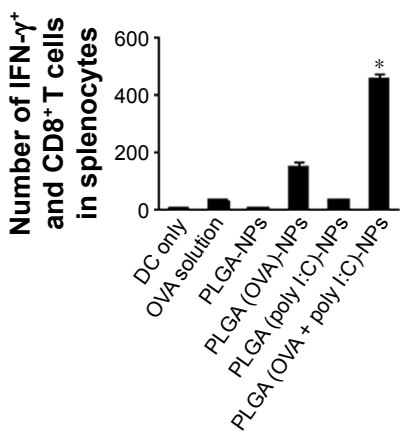

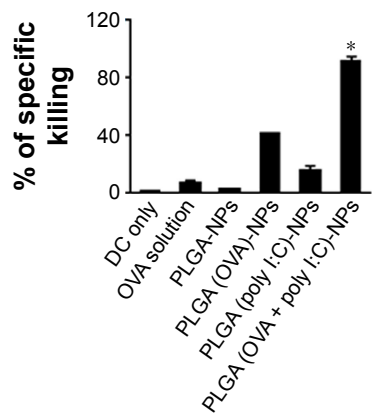

Figure 6 Vaccination with PLGA-NP-treated DCs generated an enhanced OVA-specific CD8 ${ }^{+} \mathrm{T}$ cell immune response in C57BL/6 mice (five per group). Notes: (A) OVA-specific CD8 ${ }^{+} \mathrm{T}$ cell immune responses were assessed in splenocytes of vaccinated mice by flow cytometry. The bar graph depicts the number of IFN- $\gamma+$ and OVA-specific CD8 ${ }^{+} \mathrm{T}$ cells in the splenocytes. (B) In vivo CTL assay was performed to confirm CTL response. Splenocytes were isolated from DC-vaccinated mice. OVA-specific CD8 ${ }^{+}$T cells were analyzed by flow cytometry. Bar graph depicts the percentage of specifically killed cells. Data are mean \pm SD ( $\left.n=5\right)(* P<0.0 \mathrm{I})$. Abbreviations: CFSE, carboxyfluorescein succinimidyl ester; CTL, cytotoxic T lymphocyte; DCs, dendritic cells; IFN- $\gamma$, interferon gamma; NPs, nanoparticles; OVA, ovalbumin; PLGA, poly(D,L-lactide-co-glycolide); poly I:C, polyinosinic-polycytidylic acid; SD, standard deviation.

weight, feeding habits, or behavior between the groups, suggesting that there were no overt toxicities related to the therapy. In addition, we examined the tumors for markers of anti-CD8 antibody to determine $\mathrm{CD} 8^{+} \mathrm{T}$ cells in the tumor tissue. PLGA (OVA + poly I:C)-NP-treated DCs showed a significantly higher population of $\mathrm{CD} 8^{+} \mathrm{T}$ cells in the tumor tissue compared to control or other treatment groups, and apoptosis was increased (Figure 7E).

To establish that the therapeutic effects of PLGA-NPs are not unique to just one target antigen, we also performed an in vivo experiment with additional target E7 antigens. We prepared E7 peptide (AGQAEPDRAHYNIVTFCCKCDS)incorporated PLGA (E7 + poly I:C)-NPs against the TC-1 tumor model, which expresses HPV16 and HPV-E7 proteins. Mice were randomly allocated to the following groups ( $\mathrm{n}=6$ mice/group): (1) DCs only, (2) DCs treated with E7 solution $(100 \mu \mathrm{g})$, (3) DCs treated with PLGA-NPs, (4) DCs treated with PLGA (E7)-NPs, (5) DCs treated with PLGA (poly I:C)-NPs, and (6) DCs treated with PLGA (E7 + poly I:C)-NPs. The experimental groups underwent three intraperitoneal injections at weekly intervals after 7 days of tumor cell injection (Figure 8A). Treatment with PLGA (E7 + poly I:C)-NPs resulted in significant inhibition of tumor growth as compared to DC alone (control group, $91 \%$ reduction; $P<0.01$ ), E7 solution ( $89 \%$ reduction; $P<0.01$ ), and PLGA-NPs (92\% reduction; $P<0.01$ ) (Figure $8 \mathrm{~B}$ and $\mathrm{D}$ ). Notably, $100 \%$ of mice vaccinated with DCs treated with PLGA (E7 + poly I:C)-NPs survived over 40 days, while mice vaccinated with control DCs, E7 solution, and PLGANPs died within 35 days after tumor inoculation (Figure 8C). In addition, PLGA (E7 + poly I:C)-NP-treated DCs showed significantly higher populations of $\mathrm{CD} 8^{+} \mathrm{T}$ cells in the tumor tissue compared to the other treatment groups, and apoptosis was increased (Figure 8E).

To determine $\mathrm{CD} 8^{+} \mathrm{T}$ cell-mediated immune response, we performed tumor protection experiment with antibody depletion (Figure 9). Mice were randomly allocated to the following groups ( $\mathrm{n}=6$ mice/group): (1) DCs only, (2) DCs treated with PLGA (OVA + poly I:C)-NPs with $\mathrm{CD} 8^{+} \mathrm{T}$ cell depletion, and (3) DCs treated with PLGA (poly I:C)-NPs. The experimental schedule followed for mice treatment is presented in Figure 9A. Treatment with PLGA (OVA + poly I:C)-NPs with $\mathrm{CD} 8^{+} \mathrm{T}$ cell depletion showed nearly the same volume of tumor as compared to DC alone (control group; $P<0.65$ ) (Figure 9B), and the tumor mass is shown in Figure $9 \mathrm{C}$. 
A

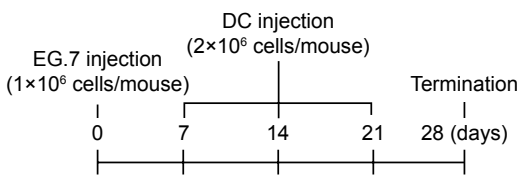

B

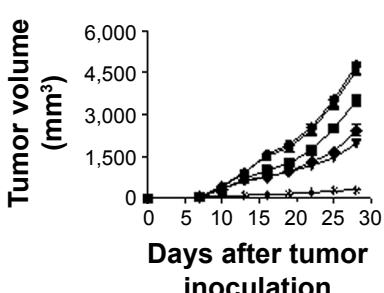

C

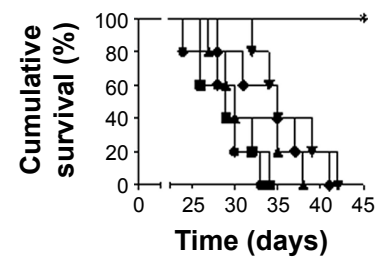

* Control (DC alone)

tr PLGA-NPs

- OVA solution

$\mp$ PLGA (OVA)-NPs

PLGA (poly I:C)-NPs * PLGA (OVA + poly I:C)-NPs

D

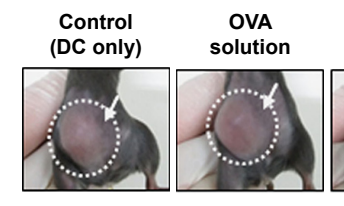

PLGA-NPs

PLGA
(OVA)-NPS

PLGA

PLGA (OVA +

(poly I:C)-NPs poly I:C)-NPs

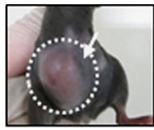

E
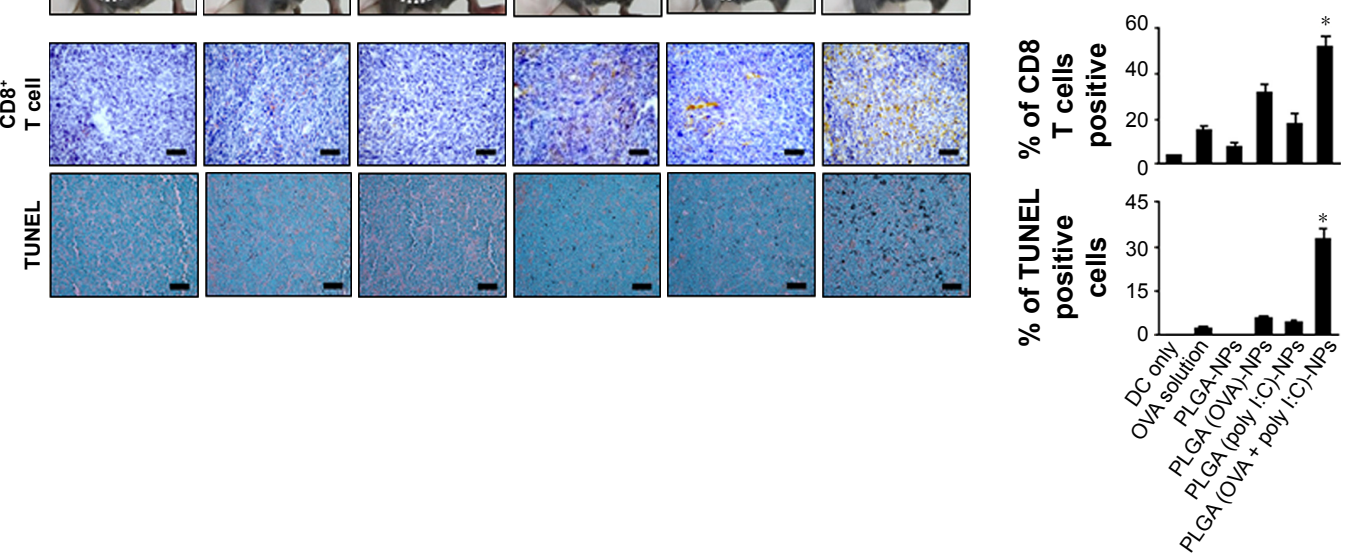

Figure 7 Antitumor efficacy of DCs treated with PLGA-NPs in the EG.7 tumor model.

Notes: Treatment was started I week after sc injection of tumor cells into mice. DCs only, DCs treated with OVA solution, DCs treated with PLGA-NPs, DCs treated with PLGA (OVA)-NPs, DCs treated with PLGA (poly I:C)-NPs, and DCs treated with PLGA (OVA + poly I:C)-NPs were administered three times weekly at a dose of $100 \mu \mathrm{g}$ of OVA and $100 \mu \mathrm{g}$ of poly I:C by sc injection. (A) Experimental schedule for DC-based tumor therapy. (B) Tumor volume after treatment with the various formulations. (C) Survival of mice. (D) Images of treated mice. (E) Immunohistochemistry for CD8 T cell localization (CD8 antibody, scale bar: $50 \mu$ m) and tumor cell apoptosis (procaspase 3 antibody, scale bar: $50 \mu \mathrm{m}$ ) were performed on EG.7-tumor tissues following vaccination with DCs. Error bars represent sem. $* P$-value (PLGA [OVA + poly I:C]-NPs) vs DC only. $P<0.05$.

Abbreviations: DCs, dendritic cells; NPs, nanoparticles; OVA, ovalbumin; PLGA, poly(D,L-lactide-co-glycolide); poly I:C, polyinosinic-polycytidylic acid; sc, subcutaneous; sem, standard error of mean; TUNEL, terminal deoxynucleotidyl transferase dUTP nick-end labeling.

\section{Discussion}

We demonstrated here that an effective PLGA-NP-based delivery system loaded with adjuvant and antigen to increase the maturation of DCs leads to potent antigen-specific CD8 ${ }^{+}$ T cell immunity in EG.7 and TC-1 tumor-bearing mice. This approach has broad utility for adjuvant delivery as well as tumor-specific antigen delivery. The PLGA-NPs were effective in delivering both adjuvant and antigen and in achieving high therapeutic efficacy.

Adjuvant and antigen delivery to DCs is a key parameter for medical and pharmaceutical applications of DC-based cancer immunotherapies to initiate the maturation of DCs. However, limited intracellular delivery of the target molecules must be overcome in order to realize the full potential of this therapeutic strategy in the clinical setting. Moreover, the delivery efficiency of free adjuvant or antigen without the use of NPs is quite low, and most of the adjuvant or antigen is rapidly degraded following injection. ${ }^{18}$ Therefore, to overcome this limitation, effective delivery systems are needed.

While a number of NP systems have been utilized for therapeutic applications, most of these systems result in wide distribution of NPs throughout the body and can lead to toxicity in normal tissues. In addition, single-adjuvant delivery may require higher doses for effective intracellular delivery to DCs. These systems include microparticles, ${ }^{31}$ nanofibers, ${ }^{32}$ metal-based particles, ${ }^{33}$ and emulsions. ${ }^{34}$ Although many types of compounds have potential utility as delivery agents, some are associated with concerns regarding their safety and evoked immune responses. The development of a DC-based vaccination for immunotherapy, therefore, requires clinically suitable, safe, and effective delivery 
A
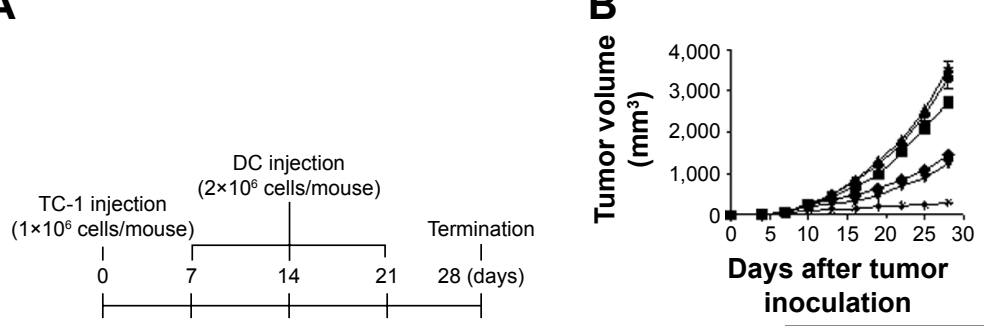

- Control (DC alone)

tr PLGA-NPS

- PLGA (poly I:C)-NPs
C

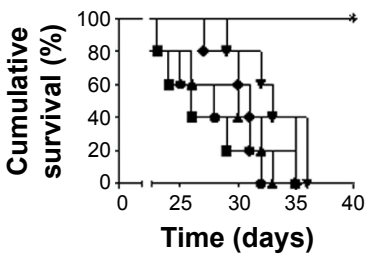

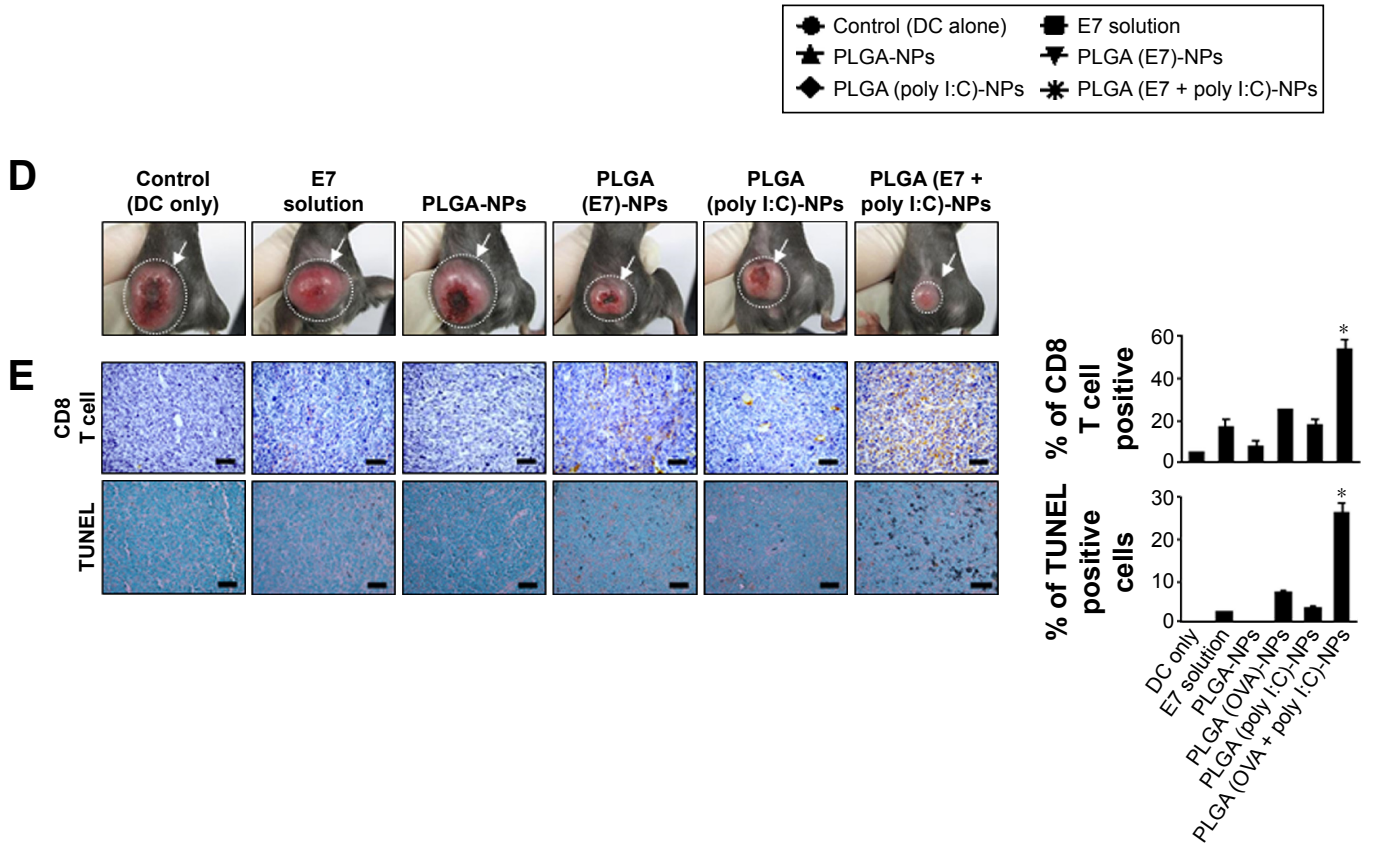

Figure 8 Antitumor efficacy of DCs treated with PLGA-NPs in the TC-I tumor model.

Notes: Treatment was started I week after sc injection of tumor cells into mice. DCs only, DCs treated with E7 solution, DCs treated with PLGA-NPs, DCs treated with PLGA (E7)-NPs, DCs treated with PLGA (poly I:C)-NPs, and DCs treated with PLGA (E7 + poly I:C)-NPs were administered three times weekly at a dose of I00 $\mu g$ of E7 and $100 \mu \mathrm{g}$ of poly I:C by sc injection. (A) Experimental schedule for DC-based tumor therapy. (B) Tumor volume after treatment with the various formulations. (C) Survival of mice. (D) Images of treated mice. (E) Immunohistochemistry for CD8 T cell localization (CD8 antibody, scale bar: $50 \mu \mathrm{m}$ ) and tumor cell apoptosis (procaspase 3 antibody, scale bar: $50 \mu \mathrm{m}$ ) were performed on EG.7-tumor tissues following vaccination with DCs. Error bars represent sem. *P-value (PLGA [OVA + poly I:C]-NPs) vs DC only. $P<0.05$

Abbreviations: DCs, dendritic cells; NPs, nanoparticles; OVA, ovalbumin; PLGA, poly(D,L-lactide-co-glycolide); poly I:C, polyinosinic-polycytidylic acid; sc, subcutaneous; sem, standard error of mean; TUNEL, terminal deoxynucleotidyl transferase dUTP nick-end labeling.

systems. NPs are promising and highly desirable for their potential to overcome the limitations of conventional compound delivery. Moreover, therapeutic payloads packaged into NPs should be a clinically viable approach for the development of vaccine-related immunotherapy.

PLGA-NPs are an attractive platform for payload delivery because of their biocompatibility, biodegradability, low toxicity, and low immunogenicity, which are key parameters for medical and pharmaceutical applications. ${ }^{13}$ Moreover, payloads are frequently loaded within the particles, and their type and number may not affect the pharmacokinetics and biodistribution of the NPs. Recent work comparing adjuvant delivery has shown that the primary role is to increase DC maturation. Here, we loaded poly I:C into PLGA-NPs as an adjuvant, which can stimulate TLR3 receptor in the endosome within the DCs, leading to DC maturation. Poly I:C is a promising immunostimulant for cancer vaccines directed against intracellular pathogens and strongly drives cellmediated immunity and type I IFN response. ${ }^{18,35}$ In addition, we demonstrated the therapeutic efficacy of DC-based immunotherapy using PLGA (OVA + poly I:C)-NPs in different tumor models and confirmed that antigen-specific $\mathrm{CD} 8^{+} \mathrm{T}$ cell activation contributed to inhibition of tumor growth.

NP-based systems have been used as effective antigen or adjuvant delivery carriers to increase penetration into DCs through ex vivo manipulation. Moreover, NP systems have also been utilized for delivery of cargo by intratumoral injection to promote immune responses in the tumor microenvironment. In this study, we established the optimum formulation to increase the coloading efficiency of antigen and adjuvant. Combinations of different antigens or, more importantly, combinations of antigens and adjuvants in the same particle can produce effective immune response. Antigens and adjuvants have to be codelivered to the same cell. 
A

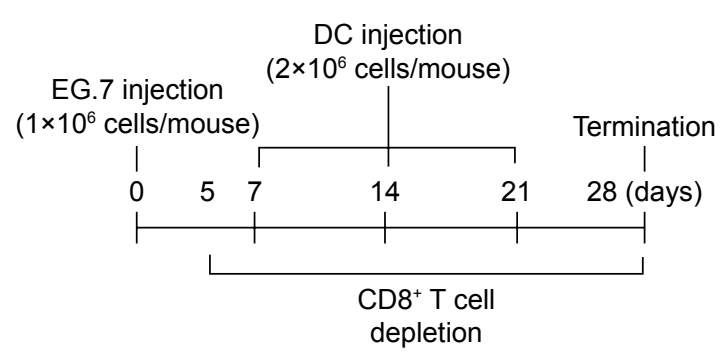

B

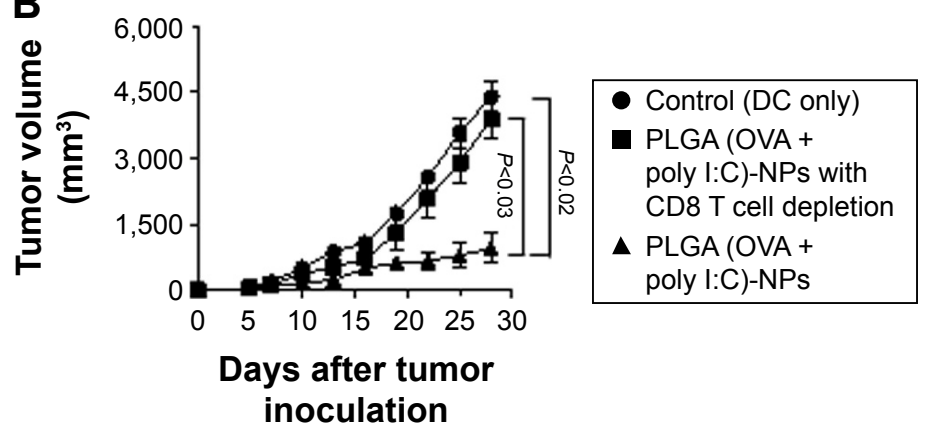

C

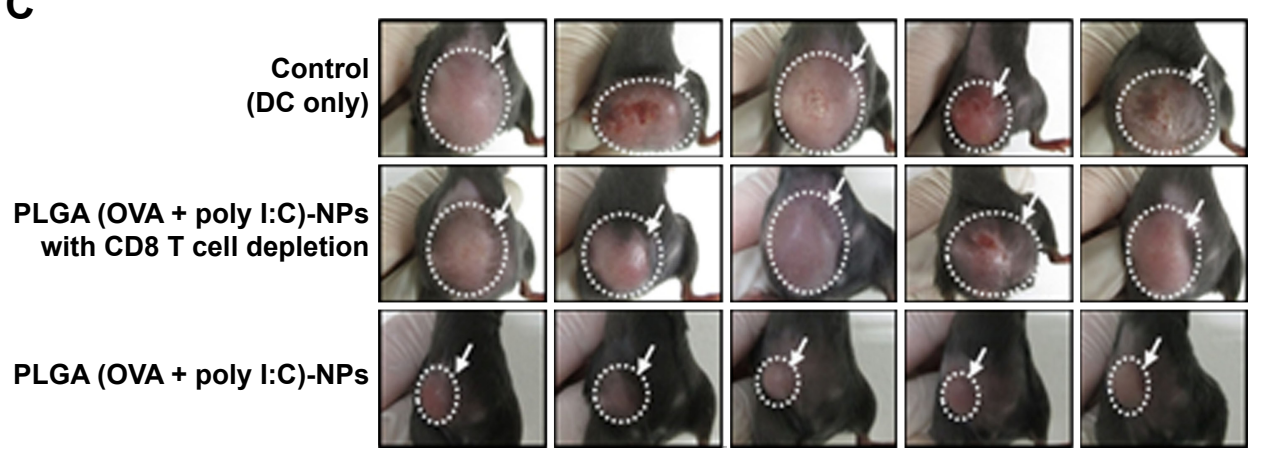

Figure 9 Antitumor efficacy of DCs treated with PLGA (OVA + poly)-NPs with CD8 ${ }^{+} \mathrm{T}$ cell depletion.

Notes: DCs were administered three times (sc, $2 \times 10^{6}$ cells/mouse, I week intervals for 3 weeks) in the right flank. Depletions (I00 $\mu g$ of anti-CD8 antibody, once daily) were initiated 5 days after tumor cell injection and lasted until the end of the experimental period. (A) Experimental schedule for DC-based tumor therapy with CD8 ${ }^{+} \mathrm{T}$ cell depletion. (B) Tumor volume. (C) Images of treated mice. Error bars represent sem.

Abbreviations: DCs, dendritic cells; NPs, nanoparticles; OVA, ovalbumin; PLGA, poly(D,L-lactide-co-glycolide); poly I:C, polyinosinic-polycytidylic acid; sc, subcutaneous; sem, standard error of mean.

PLGA (OVA + poly I:C)-NPs with high coloading efficiency and containing low doses of antigens and adjuvants were able to induce strong DC maturation and antigen-specific activation. The use of lower doses in our system would be advantageous not only for minimizing the potential side effects associated with the use of adjuvants, but also from an economical point of view. In addition, we demonstrated the therapeutic efficacy of DC-based cancer immunotherapy in different tumor models. PLGA (antigen + poly I:C) treatment of DCs in vitro resulted in antigen-specific DC maturation. After injection of mature DC into tumor-bearing mice, we confirmed antigen-specific $\mathrm{CD} 8^{+} \mathrm{T}$ cell activation leading to increased therapeutic efficacy.

DC-based cancer immunotherapy for adjuvant and/or antigen delivery leads to potent immune responses in mice. This study provides a rational approach to improving the safety and reducing the toxicity of adjuvants. In addition, the NP system allows for the codelivery of therapeutic payloads, such as an antigen or adjuvant, which could further enhance the antigen-specific immune response without increasing toxicity. This NP-based adjuvant delivery system may be attractive for many diverse biomedical applications. Although the PLGA-NP system could be useful for diseases associated with the immune system and for enhancing the immune responses, additional possibilities for immune modulation using the PLGA-NP platform may be explored and developed for research purposes.

\section{Conclusion}

We demonstrated the ability of the PLGA-NP platform to enhance the potency of DC-based vaccination in tumor-bearing mice. We showed that PLGA (OVA + poly I:C)-NPs induced DC activation and maturation. Furthermore, PLGA (OVA + poly I:C)-NP-treated DCs pulsed with OVA peptides generated higher numbers of OVA-specific $\mathrm{CD} 8^{+} \mathrm{T}$ cells, which led to potent antitumor efficacy in EG.7 and TC-1 tumorbearing mice. In addition, the antigen-specific $\mathrm{CD} 8^{+} \mathrm{T}$ cell immune response elicited by the PLGA (OVA + poly I:C)NP-treated DC vaccine led to more potent antitumor effects and prolonged survival in tumor-bearing mice. This PLGANP-based delivery system will also be useful for delivering many other target adjuvants or antigens to DCs. In addition, the PLGA-NP system could be expanded and developed to include additional therapeutic and experimental approaches. The PLGA-NP-based strategy presented here has broad potential as a delivery platform for increasing the immune responses to DC-based cancer immunotherapy and could be adapted for other immune-associated diseases. 


\section{Acknowledgments}

This work was supported by the National Research Foundation of Korea (NRF) grant funded by the Korean government (NRF-2015R1A2A1A13001713, NRF-2016R1A5A2012284, NRF-2015R1A2A2A04003620) (Y-MP and YJL). This work was supported by Basic Research Laboratory Program through the NRF funded by the Ministry of Science (NRF2016R1A2B2007327), and ICT and Future Planning (No 2013R1A4A1069575) (HDH). This study was supported by a grant from the National R\&D program for Cancer Control, Ministry for Health, Welfare and Family affairs, Republic of Korea (1520100) (J-WL, and HDH). The authors would like to express their thanks to Ga Hee Kim, Min Gi Kim, and Hyung Jun Ahn for data analysis.

\section{Disclosure}

The authors report no conflicts of interest in this work.

\section{References}

1. Heo MB, Cho MY, Lim YT. Polymer nanoparticles for enhanced immune response: combined delivery of tumor antigen and small interference RNA for immunosuppressive gene to dendritic cells. Acta Biomater. 2014;10(5):2169-2176.

2. Hubbell JA, Thomas SN, Swartz MA. Materials engineering for immunomodulation. Nature. 2009;462(7272):449-460.

3. Steinman RM. Dendritic cells in vivo: a key target for a new vaccine science. Immunity. 2008;29(3):319-324.

4. Gelao L, Criscitiello C, Esposito A, et al. Dendritic cell-based vaccines: clinical applications in breast cancer. Immunotherapy. 2014;6(3): 349-360.

5. Chiang CL, Balint K, Coukos G, Kandalaft LE. Potential approaches for more successful dendritic cell-based immunotherapy. Expert Opin Biol Ther. 2015;15(4):569-582.

6. Cruz LJ, Rosalia RA, Kleinovink JW, Rueda F, Lowik CW, Ossendorp F. Targeting nanoparticles to CD40, DEC-205 or CD11c molecules on dendritic cells for efficient CD8(+) T cell response: a comparative study. J Control Release. 2014;192:209-218.

7. Reddy ST, Swartz MA, Hubbell JA. Targeting dendritic cells with biomaterials: developing the next generation of vaccines. Trends Immunol. 2006; 27(12):573-579.

8. Rosalia RA, Silva AL, Camps M, et al. Efficient ex vivo induction of $T$ cells with potent anti-tumor activity by protein antigen encapsulated in nanoparticles. Cancer Immunol Immunother. 2013;62(7):1161-1173.

9. Liu SY, Wei W, Yue H, et al. Nanoparticles-based multi-adjuvant whole cell tumor vaccine for cancer immunotherapy. Biomaterials. 2013;34(33): 8291-8300.

10. Felice B, Prabhakaran MP, Rodriguez AP, Ramakrishna S. Drug delivery vehicles on a nano-engineering perspective. Mater Sci Eng C Mater Biol Appl. 2014;41:178-195.

11. van der Meel R, Vehmeijer LJ, Kok RJ, Storm G, van Gaal EV. Ligandtargeted particulate nanomedicines undergoing clinical evaluation: current status. Adv Drug Deliv Rev. 2013;65(10):1284-1298.

12. Godsey ME, Suryaprakash S, Leong KW. Materials innovation forco-delivery of diverse therapeutic cargos. RSC Adv. 2013;3(47):24794-24811.

13. Joshi VB, Geary SM, Salem AK. Biodegradable particles as vaccine antigen delivery systems for stimulating cellular immune responses. Hum Vaccin Immunother. 2013;9(12):2584-2590.

14. Heo MB, Lim YT. Programmed nanoparticles for combined immunomodulation, antigen presentation and tracking of immunotherapeutic cells. Biomaterials. 2014;35(1):590-600.
15. Zhang Z, Tongchusak S, Mizukami Y, et al. Induction of anti-tumor cytotoxic T cell responses through PLGA-nanoparticle mediated antigen delivery. Biomaterials. 2011;32(14):3666-3678.

16. Perez-Giron JV, Belicha-Villanueva A, Hassan E, et al. Mucosal polyinosinic-polycytidylic acid improves protection elicited by replicating influenza vaccines via enhanced dendritic cell function and $\mathrm{T}$ cell immunity. J Immunol. 2014;193(3):1324-1332.

17. Quinn KM, Yamamoto A, Costa A, et al. Coadministration of polyinosinic:polycytidylic acid and immunostimulatory complexes modifies antigen processing in dendritic cell subsets and enhances HIV gag-specific T cell immunity. J Immunol. 2013;191(10):5085-5096.

18. Hafner AM, Corthesy B, Merkle HP. Particulate formulations for the delivery of poly (I:C) as vaccine adjuvant. Adv Drug Deliv Rev. 2013; 65(10):1386-1399.

19. Yao MH, Ma M, Chen Y, et al. Multifunctional Bi2S3/PLGA nanocapsule for combined HIFU/radiation therapy. Biomaterials. 2014;35(28): 8197-8205.

20. Zhang Y, Gou J, Sun F, et al. Impact of electrolytes on double emulsion systems $(\mathrm{W} / \mathrm{O} / \mathrm{W})$ stabilized by an amphiphilic block copolymer. Colloids Surf B Biointerfaces. 2014;122:368-374.

21. Dolen Y, Kreutz M, Gileadi U, et al. Co-delivery of PLGA encapsulated invariant NKT cell agonist with antigenic protein induce strong T cell-mediated antitumor immune responses. Oncoimmunology. 2015; 5(1):e1068493.

22. Zhou F, Ciric B, Zhang GX, Rostami A. Immunotherapy using lipopolysaccharide-stimulated bone marrow-derived dendritic cells to treat experimental autoimmune encephalomyelitis. Clin Exp Immunol. 2014;178(3):447-458.

23. Roh JW, Huang J, Hu W, et al. Biologic effects of platelet-derived growth factor receptor alpha blockade in uterine cancer. Clin Cancer Res. 2014;20(10):2740-2750.

24. Kang TH, Kim YS, Kim S, et al. Pancreatic adenocarcinoma upregulated factor serves as adjuvant by activating dendritic cells through stimulation of TLR4. Oncotarget. 2015;6(29):27751-27762.

25. Kang TH, Knoff J, Yeh WH, et al. Treatment of tumors with vitamin E suppresses myeloid derived suppressor cells and enhances $\mathrm{CD} 8^{+}$ T cell-mediated antitumor effects. PLoS One. 2014;9(7):e103562.

26. Jung ID, Shin SJ, Lee MG, et al. Enhancement of tumor-specific $\mathrm{T}$ cell-mediated immunity in dendritic cell-based vaccines by Mycobacterium tuberculosis heat shock protein X. J Immunol. 2014;193(3): 1233-1245.

27. Song CK, Han HD, Noh KH, et al. Chemotherapy enhances CD8(+) $\mathrm{T}$ cell-mediated antitumor immunity induced by vaccination with vaccinia virus. Mol Ther. 2007;15(8):1558-1563.

28. Ramakrishnan R, Assudani D, Nagaraj S, et al. Chemotherapy enhances tumor cell susceptibility to CTL-mediated killing during cancer immunotherapy in mice. J Clin Invest. 2010;120(4):1111-1124.

29. Cho MS, Vasquez HG, Rupaimoole R, et al. Autocrine effects of tumorderived complement. Cell Rep. 2014;6(6):1085-1095

30. Carmi Y, Spitzer MH, Linde IL, et al. Allogeneic IgG combined with dendritic cell stimuli induce antitumour T-cell immunity. Nature. 2015; 521(7550):99-104.

31. Singh M, Chakrapani A, O'Hagan D. Nanoparticles and microparticles as vaccine-delivery systems. Expert Rev Vaccines. 2007;6(5):797-808.

32. Chen J, Pompano RR, Santiago FW, et al. The use of self-adjuvanting nanofiber vaccines to elicit high-affinity B cell responses to peptide antigens without inflammation. Biomaterials. 2013;34(34):8776-8785.

33. Yu X, Feizpour A, Ramirez NG, et al. Glycosphingolipid-functionalized nanoparticles recapitulate CD169-dependent HIV-1 uptake and trafficking in dendritic cells. Nat Commun. 2014;5:4136.

34. Zeng BJ, Chuan YP, O'Sullivan B, et al. Receptor-specific delivery of protein antigen to dendritic cells by a nanoemulsion formed using top-down non-covalent click self-assembly. Small. 2013;9(22):3736-3742.

35. Ammi R, De Waele J, Willemen Y, et al. Poly(I:C) as cancer vaccine adjuvant: knocking on the door of medical breakthroughs. Pharmacol Ther. 2015;146:120-131. 


\section{Supplementary materials}

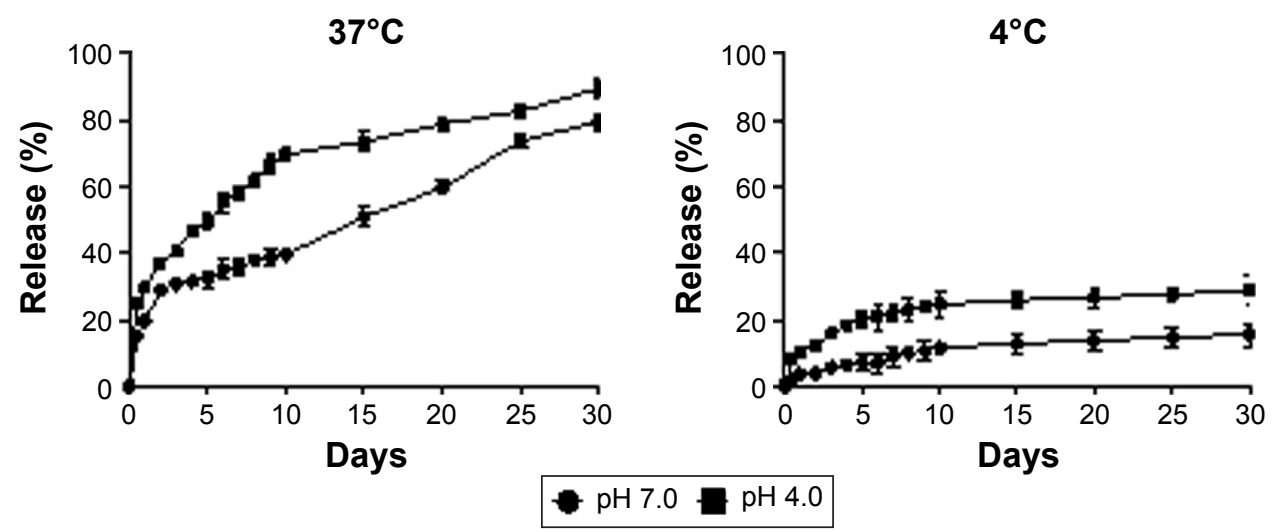

Figure SI Release behavior of OVA from PLGA (OVA + poly I:C)-NP.

Note: The data are represented as mean \pm SD $(n=3)$.

Abbreviations: NPs, nanoparticles; OVA, ovalbumin; PLGA, poly(D,L-lactide-co-glycolide); poly I:C, polyinosinic-polycytidylic acid; SD, standard deviation.
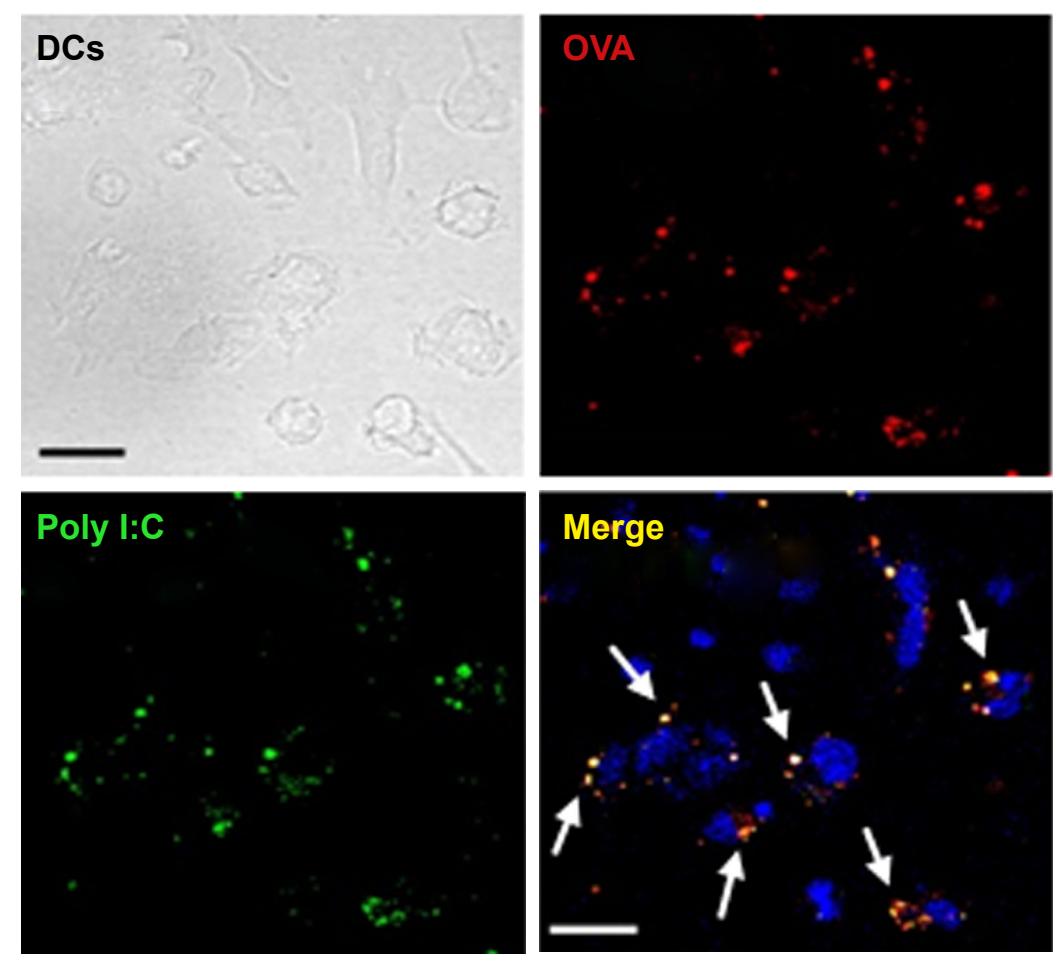

Figure S2 Intracellular delivery of PLGA (OVA + poly I:C)-NP in DCs. OVA was labeled with TRICT fluorescence and poly I:C labeled with FITC. Scale bar: $20 \mu$ m. Abbreviations: DCs, dendritic cells; FITC, fluorescein isothiocyanate; NPs, nanoparticles; OVA, ovalbumin; PLGA, poly(D,L-lactide-co-glycolide); poly I:C, polyinosinicpolycytidylic acid; TRITC, Tetramethylrhodamine.

\section{Publish your work in this journal}

The International Journal of Nanomedicine is an international, peerreviewed journal focusing on the application of nanotechnology in diagnostics, therapeutics, and drug delivery systems throughout the biomedical field. This journal is indexed on PubMed Central, MedLine, CAS, SciSearch $®$, Current Contents ${ }^{\circledR} /$ Clinical Medicine,
Journal Citation Reports/Science Edition, EMBase, Scopus and the Elsevier Bibliographic databases. The manuscript management system is completely online and includes a very quick and fair peer-review system, which is all easy to use. Visit http://www.dovepress.com/ testimonials.php to read real quotes from published authors. 